\title{
Tumor Infiltrating Lymphocytes - The Next Step in Assessing Outcome and Response to Treatment in Patients with Breast Cancer
}

\section{Robert Wesolowski ${ }^{{ }^{*}}$ and William E Carson III ${ }^{2}$}

${ }^{1}$ Center of Internal Medicine, Division of Medical Oncology, Ohio State University, Columbus, USA

${ }^{2}$ Department of Surgical Oncology; OSU Comprehensive Cancer Center, Ohio State University, Columbus, USA

"Corresponding author: Robert Wesolowski, Assistant Professor of Internal Medicine, Division of Medical Oncology, B401 Starling Loving Hall, W10th Avenue, Columbus, OH 44210, USA, Tel: (614) 366-4810; Fax: (614) 293 - 4372; E-mail: Robert.Wesolowski@osumc.edu

Received date: Oct 15, 2014, Accepted date: Nov 20, 2014, Published date: Nov 25, 2014

Copyright: (c) 2014 Wesolowski R, et al. This is an open-access article distributed under the terms of the Creative Commons Attribution License, which permits unrestricted use, distribution, and reproduction in any medium, provided the original author and source are credited.

\section{Abstract}

Tumor infiltrating lymphocytes are studied for their potential as new clinically useful prognostic and predictive biomarkers in patients with triple negative and HER-2/neu amplified breast cancer. This area of research could also help guide the development of novel therapeutic approaches for these diseases.

Keywords: Breast cancer; Tumor infiltrating lymphocytes; Biomarkers

\section{Introduction}

Over the recent years, new results from multiple groups have pointed towards Tumor Infiltrating Lymphocytes (TILs) as prognostic and predictive biomarkers in breast cancer. This is not a new concept. For instance, it has been known since 1940s that a subtype of breast cancer that characteristically exhibits a very large proportion of stromal lymphocytic infiltrate, called medullary carcinoma, has been associated with excellent prognosis after aggressive local therapy in spite of high histologic grade and axillary lymph node metastases $[1,2]$. However, over the next several decades, a number of studies examined the association between tumor lymphocytic infiltrates and prognosis in more common histologic types of breast cancer and yielded contradictory results [3-6]. The concept of the immune tumor microenvironment's role in influencing the outcomes of breast cancer patients has resurfaced after the recognition that expression of genes associated with stromal (fibroblast) and immune (macrophage, T-cell) components significantly contributes to the global gene expression landscape within the breast tumors as detected by the use of cDNA based gene expression microarrays [7]. High expression of genes typical for lymphocytes (such as CD8) and low expression of genes typical of myeloid cells (such as CD68) was found to indicate better prognosis, especially in basal like and HER-2/neu positive breast cancer subtypes [8-10]. This has generated a renewed excitement and multiple investigators began to study the potential association between TILs, prognosis and response to systemic therapy for breast cancer. Lymphocytes can be easily detected by analyzing slides of tumor sections stained with hematoxylin and eosin ( $\mathrm{H} \& \mathrm{E})$; a simple and inexpensive technique that can be reliably performed by practically any pathologist.

\section{Tumor Infiltrating Lymphocytes and Adjuvant Chemotherapy Trials}

To study the association between TILs (detected on H\&E stain) and survival, investigators utilized tumors collected from breast cancer patients who participated in randomized clinical trials of adjuvant chemotherapy. The advantage of this approach is the availability of samples from large cohorts of patients with abundance of data on clinical characteristics of their disease and their outcomes. By using such an approach, Loi and colleagues have demonstrated that a high proportion of TILs $(>50 \%)$ in tumors of women with operable, triple negative breast cancer enrolled in a large adjuvant chemotherapy trial called BIG 2-98, was strongly associated with favorable disease free $(\mathrm{p}=0.014)$ and overall $(\mathrm{p}=0.029)$ survival [11]. In their analysis, two distinct subsets of lymphocytes were measured, namely (1) stromal TILs (sTILs - mononuclear cells that were present within the tumor stroma but were not in direct contact with invasive carcinoma cells) and (2) intratumoral TILs (iTILs - mononuclear cells that were directly associated with the malignant cells). Their analysis revealed that for every $10 \%$ increase in the levels of sTILs there was $17 \%$ reduction in the risk of relapse $(\mathrm{p}=0.025)$ and death $(\mathrm{p}=0.023)$. They also found close association between higher levels of sTILs and iTILs with infiltrating ductal histology $(\mathrm{P}<0.001$ and 0.48 respectively), high histologic grade (both $\mathrm{p}<0.001$ ), hormone receptor negativity (both $\mathrm{p}<0.001)$, and increased Ki67 expression $(>14 \%$; both $\mathrm{p}<0.001)$. These results suggest that perhaps lymphocyte predominance could select a subgroup of patients with favorable prognosis despite having other poor clinical and histologic characteristics. No association was found between TIL levels and outcomes of patients with hormone receptor positive or HER-2/neu amplified breast cancer. In the recently presented confirmatory study, tumor samples of women with operable triple negative breast cancer from two large adjuvant trials (ECOG 2197 and ECOG 1199) were analyzed for the presence of TILs [12]. Again, the investigators found that for every $10 \%$ increase in sTILs there was $18 \%$ reduction in the risk of distant recurrence and $19 \%$ reduction in the risk of death. The presence of iTILs showed a trend towards better outcome but it did not reach statistical significance. On multivariate analysis, high levels of sTILs predicted improved disease free, distant recurrence free and overall survival independently of other poor clinical and histologic characteristics such as tumor size, lymph node metastases or patient age. Similar findings were shown in patients with triple negative and HER-2/neu positive breast cancer enrolled on a phase III, randomized FinHER trial that tested different adjuvant chemotherapy regiments with or without trastuzumab. This analysis also suggested for the first time that higher levels of TILs 
could be associated with increased trastuzumab benefit in HER-2/neu positive breast cancer [13].

\section{Tumor Infiltrating Lymphocytes and Neo-adjuvant Chemotherapy Trials}

Other studies looked at association between levels of TILs and the likelihood of achieving a complete pathologic response (pCR) following neo-adjuvant chemotherapy for breast cancer. Complete pathologic response is a surrogate biomarker that is generally considered to indicate a lower likelihood of recurrence and a higher chance of survival [14]. Loi and colleagues analyzed biopsy specimen from 156 patients with HER-2/neu positive breast cancer enrolled in GeparQuatro trial and treated with trastuzumab containing neoadjuvant chemotherapy [15]. In this study, every $10 \%$ increase in the levels of TILs resulted in 16\% increase in the likelihood of achieving pCR. Forty seven percent of patients with high levels of TILs achieved pCR in contrast to only $31.7 \%$ of patients in the entire cohort. This provided additional support to the notion that TIL levels might correlate with the response to trastuzumab based chemotherapy. In another analysis of tumor samples from patients enrolled in a neoadjuvant Neo-Sphere trial that tested neo-adjuvant pertuzumab, trastuzumab, or both, with or without chemotherapy, Gianni and colleagues discovered that high expression of immune checkpoints within breast tumors, such as PD1 and PDL1 (which inhibit the function of T-lymphocytes) were associated with lower likelihood of achieving pCR after chemotherapy in combination with trastuzumab and pertuzumab [16]. Other groups have found a correlation between levels of TILs and response to carboplatin. In an analysis of 580 diagnostic tumor biopsies obtained from patients on a clinical trial testing neo-adjuvant chemotherapy with or without carboplatin (Gepar Sixto trial), approximately $60 \%$ of patients with lymphocyte predominant breast cancer (defined as presence of $\geq 60 \%$ of TILs) achieved pCR compared to pCR rate of $40 \%$ in all participants and $34 \%$ in patients with low levels of TILs $(p<0.0005)$ [17]. Among patients with high levels of TILs, those who received carboplatin achieved a pCR rate of $74 \%$ compared to $46.6 \%$ in patients treated without carboplatin. A similar study by Vinayak and colleagues analyzed diagnostic biopsies of patients with triple negative breast cancer treated with neo-adjuvant carboplatin, gemcitabine and a Poly (ADP - Ribose) Polymerase (PARP) inhibitor iniparib [18]. In this analysis, tumors with high levels of TILs were tightly correlated with an immunomodulatory subtype of triple negative breast cancer, based on Vanderbilt classification [19]. There was a strong association between high levels of both sTIL and iTIL and residual tumor burden score (an objective method of assessing pathologic response to neoadjuvant chemotherapy). However, an association remained statistically significant only for iTILs after adjustments for age, tumor size, $\mathrm{N}$ stage, tumor grade and germ line BRCA status. For every $10 \%$ increase in iTILs there was a $162 \%$ increase in the odds of achieving pCR.

\section{Clinical Significance and Future Directions}

What are the implications of these findings to current clinical practice? Perhaps, the results of the above studies could lead to the routine practice of reporting the levels of lymphocytic infiltrates in the pathology reports of patients diagnosed with breast carcinoma (especially in cases of triple negative and HER-2/neu positive disease)? Before that occurs, the methodology of interpreting TILs and the cut off values for lymphocyte predominant breast cancer will need to be standardized. This data will also require future validation by other groups in prospectively designed clinical trials with pre-specified immune correlative endpoints. Regardless, the findings from the above analysis are already providing crucial clues for future clinical research. As an example, these studies corroborate pre-clinical work by others suggesting that perhaps some conventional anti-neoplastic drugs such as platinum agents or trastuzumab could induce anti-tumor responses at least in part by modulating the host immune system [13,17,20-23]. The finding that high expression of immune checkpoints is associated with lower pCR rates after neo-adjuvant therapy with monoclonal antibodies against HER-2/neu, provides support for studying combinations of trastuzumab and/or pertuzumab and PD1, PDL1 or CTLA-4 inhibitors. Additionally, since high levels of TILs are most common in the immune-modulatory subtype of triple negative breast cancer, it may be reasonable to select such patients for treatment with immune based cancer therapies. Another important issue that is currently being studied is whether predominance of different subtypes of intra-tumoral lymphocytes (such as CD4+ T-helper cells, CD8+ cytotoxic T-lymphocytes or CD25+/FOXP3+ T-regulatory cells) could have differential effect on patient outcome. This requires more complex analyses using immunohistochemical stains, fluorescent microscopy, flow cytometry or gene expression microarrays. One such method called Cibersort is a gene expression based assay that can identify 23 leukocyte subsets present in the tumor based on a unique gene expression signature of these cells. By using Cibersort, Vinayak et al. demonstrated that high levels of activated CD4+ memory T-cells were significantly associated with pathologic response to neo-adjuvant carboplatin containing chemotherapy [18]. One more critical question is why some breast cancer patients have high levels of TILs while others don't. Understanding this may provide clues for new effective therapeutic strategies. In conclusion, the work summarized above could, in the near future, lead us towards developing vital prognostic and predictive biomarkers that would guide the selection of the most effective management strategies for breast cancer and provide support for the development of novel treatments [24].

\section{Funding}

RW is supported by Translational Grant K12 CA 133250 in experimental therapeutics from the National Cancer Institute.

\section{References:}

1. Moore Os Jr, Foote Fw Jr (1949) The relatively favorable prognosis of medullary carcinoma of the breast. Cancer 2: 635-642.

2. Richardson WW (1956) Medullary carcinoma of the breast; a distinctive tumour type with a relatively good prognosis following radical mastectomy. Br J Cancer 10: 415-423.

3. Horst HA, Horny HP (1988) Frequency distribution of lymphoreticular infiltrates in invasive carcinoma of the female breast. Cancer Detect Prev 11: $297-301$

4. Aaltomaa S, Lipponen P, Eskelinen M, Kosma VM, Marin S, et al. (1992) Lymphocyte infiltrates as a prognostic variable in female breast cancer. Eur J Cancer 28A: 859-864.

5. Chin Y, Janseens J, Vandepitte J, Vandenbrande J, Opdebeek L, et al. (1992) Phenotypic analysis of tumor-infiltrating lymphocytes from human breast cancer. Anticancer Res 12: 1463-1466.

6. Ménard S, Tomasic G, Casalini P, Balsari A, Pilotti S, et al. (1997) Lymphoid infiltration as a prognostic variable for early-onset breast carcinomas. Clin Cancer Res 3: 817-819.

7. Perou CM, Sørlie T, Eisen MB, van de Rijn M, Jeffrey SS, et al. (2000) Molecular portraits of human breast tumours. Nature 406: 747-752. 
Citation: Wesolowski R and Carson WE (2014) Tumor Infiltrating Lymphocytes - The Next Step in Assessing Outcome and Response to Treatment in Patients with Breast Cancer. J Carcinog Mutagen 5: 199. doi:10.4172/2157-2518.1000199

Page 3 of 3

8. Rody A, Holtrich U, Pusztai L, Liedtke C, Gaetje R, et al. (2009) T-cell metagene predicts a favorable prognosis in estrogen receptor-negative and HER2-positive breast cancers. Breast Cancer Res 11: R15.

9. DeNardo DG, Brennan DJ, Rexhepaj E, Ruffell B, Shiao SL, et al. (2011) Leukocyte complexity predicts breast cancer survival and functionally regulates response to chemotherapy. Cancer Discov 1: 54-67.

10. Mahmoud SM, Paish EC, Powe DG, Macmillan RD, Grainge MJ, et al. (2011) Tumor-infiltrating CD8+ lymphocytes predict clinical outcome in breast cancer. J Clin Oncol 29: 1949-1955.

11. Loi S, Sirtaine N, Piette F, Salgado R, Viale G, et al. (2013) Prognostic and predictive value of tumor-infiltrating lymphocytes in a phase III randomized adjuvant breast cancer trial in node-positive breast cancer comparing the addition of docetaxel to doxorubicin with doxorubicinbased chemotherapy: BIG 02-98. J Clin Oncol 31: 860-867.

12. Adams S, Gray RJ, Demaria S, Goldstein L, Perez EA, et al. (2014) Prognostic Value of Tumor-Infiltrating Lymphocytes in Triple-Negative Breast Cancers From Two Phase III Randomized Adjuvant Breast Cancer Trials: ECOG 2197 and ECOG 1199. J Clin Oncol .

13. Loi S, Michiels S, Salgado R, Sirtaine N, Jose V, et al. (2014) Tumor infiltrating lymphocytes are prognostic in triple negative breast cancer and predictive for trastuzumab benefit in early breast cancer: results from the FinHER trial. Ann Oncol 25: 1544-1550.

14. Mieog JS, van der Hage JA, van de Velde CJ (2007) Preoperative chemotherapy for women with operable breast cancer. Cochrane Database Syst Rev : CD005002.

15. Loi S, Michiels S, Salgado R, Sirtaine N, Jose V et al. (2013) Abstract S1-05: Tumor infiltrating lymphocytes (TILs) indicate trastuzumab benefit in early-stage HER2-positive breast cancer (HER2+ BC). Cancer Res: S1-05.

16. Gianni L, Bianchini G, Valagussa P, Belousov A, et al. (2012) Adaptive immune system and immune checkpoints are associated with response to pertuzumab $(\mathrm{P})$ and trastuzumab $(\mathrm{H})$ in the NeoSphere study Cancer Res: S6-7.

17. Denkert C, Loibl S, Salat C, Sinn BV, Schem C, et al. Increased tumorassociated lymphocytes predict benefit from addition of carboplatin to neoadjuvant therapy for triple-negative and HER2-positive early breast cancer in the GeparSixto trial (GBG 66). Cancer Res: S1-06.

18. Vinayak H, Gray RJ, Adams S, Jensen KC, Manola J, et al. (2014) Association of increased TILs with immunomodulatory (IM) triplenegative breast cancer subtype and response to neo-adjuvant platinumbased therapy in PrECOG 0105. J Clin Oncol.

19. Masuda H, Baggerly KA, Wang Y, Zhang Y, Gonzalez-Angulo AM, et al. (2013) Differential response to neoadjuvant chemotherapy among 7 triple-negative breast cancer molecular subtypes. Clin Cancer Res 19: 5533-5540.

20. Wu X, Feng QM, Wang Y, Shi J, Ge HL, et al. (2010) The immunologic aspects in advanced ovarian cancer patients treated with paclitaxel and carboplatin chemotherapy. Cancer Immunol Immunother 59: 279-291.

21. Clynes RA, Towers TL, Presta LG, Ravetch JV (2000) Inhibitory Fc receptors modulate in vivo cytotoxicity against tumor targets. Nat Med 6: 443-446.

22. McDonnell AM, Nowak AK, Lake RA (2011) Contribution of the immune system to the chemotherapeutic response. Semin Immunopathol 33: 353-367.

23. de Biasi AR, Villena-Vargas J, Adusumilli PS (2014) Cisplatin-Induced Antitumor Immunomodulation: A Review of Preclinical and Clinical Evidence. Clin Cancer Res 20: 5384-5391.

24. Melichar B, Študentova H, Kalabova H, Vitaskova D, Aceermakova P, et al. (2014) Predictive and prognostic significance of tumor-infiltrating lymphocytes in patients with breast cancer treated with neoadjuvant systemic therapy. Anticancer Res 34: 1115-1125. 